\title{
Scalp arteriovenous malformation: A case report with review
}




\title{
Scalp arteriovenous malformation: A case report with review
}

\author{
Asheesh Kumar Gupta, S.N. Iyengar, Anand Sharma
}

Department of Neurosurgery, G.R. Medical College Gwalior, INDIA

\begin{abstract}
Arteriovenous malformation (AVM) of the scalp is a rare lesion whose natural history remains to be elucidated. Clinical symptoms, usually, range from an asymptomatic lesion, local discomfort, headaches to necrosis and massive hemorrhage. Selective angiography remains the cornerstone for investigation. Complete surgical excision, embolization or an approach combining the modalities is curative. It can also cause massive hemorrhages due to dryness of the overlying skin and injuries. In this report we describe the clinical and radiological features of a patient with a scalp arteriovenous malformation.
\end{abstract}

Key words: AVM, STA, NIDUS

\section{Introduction}

Arteriovenous malformation (AVM) of the scalp is a rare vascular lesion that is characterised by abnormal fistulous connections between supplying arteries and draining veins without an intervening capillary network in the subcutaneous plane of the scalp.

(1) The scalp AVM comprises of $8.1 \%$ of total AVM and more common in young adults. A well-known fact states their origin attributable to anomalous primitive fistulous connection between feeding arteries and draining veins, with the absence of intervening capillary bed. This state of high shunt flow between the two distinct vessel types and the complex vascular anatomy makes their treatment a challenging undertaking.
We present one case of scalp AVM with review of literature.

\section{Case report}

A 20 year female presented to Neurosurgery OPD with painless swelling over left fronto-parietal region of scalp since birth increasing in size for the past 1-2 years. Initially, it was small reddish swelling till adolescent and become larger in size and causing esthetic problem to her. On examination, there was a soft, bluish and nontender lesion in the left fronts-parietal region of scalp. The overlying skin is coarse and dry; however there was neither ulceration nor active haemorrhage present (Figure 1). There was no previous history of trauma or head injury. Bruit was heard on auscultation of 
the lesion. The vital signs were stable and all other systems were normal.

Ultrasound showed mixed echoic lesions in scalp soft tissue planes which are supplied by dilated, tortuous (Serpigenous) vessels. Color Doppler revealed high velocity low resistance arterial flow is seen. Non contrast CT head revealed soft tissue component with mild tortuously in the extracranial soft tissue scalp anteriorly on left side.

Underlying bones are normal. No intracranial communication seen (Figure 2). MR Angiography showed high flow vascular malformation comprises multiple tortuous and dilated extra cranial arcade in the scalp particularly at the high frontal and vertex region with feeders coming from the branches of bilateral superficial temporal, occipital arteries, branches of external carotid arteries and supraorbital arteries, branches of facial arteries forming small nidus and vinous drainage in to scalp veins (Figure 3). After discussion of risk and benefits patient underwent gross total excision of scalp AVP. The pathological diagnosis of the patient was consistent with AVM. The histopathological specimen contained various well-developed arteries and dilated veins in the connecting tissue. The patient was discharged with no postoperative problems.

\section{Discussion}

AVM of the scalp is an uncommon entity. $(9,10)$ The vascular malformation of the scalp is an abnormal arteriovenous communication situated within the subcutaneous fatty layer of the scalp with the feeding arteries derived from the vessels supplying the scalp.
Various names are used to describe the vascular malformations of the scalp, including aneurysm cirsoides, aneurysm serpentinum, plexiform angioma, arteriovenous fistula, and AVM. The most frequent sites of involvement are frontal, temporal, and parietal regions. (11, $12,13,14)$ The origin of the main feeder is in the subcutaneous tissue of the scalp. The origin of these main feeders, most frequently, arises from the external carotid, occipital, and supraorbital arteries. The superficial temporal artery is frequently involved in traumatic cirsoid aneurysm due to its long unprotected course. (4)

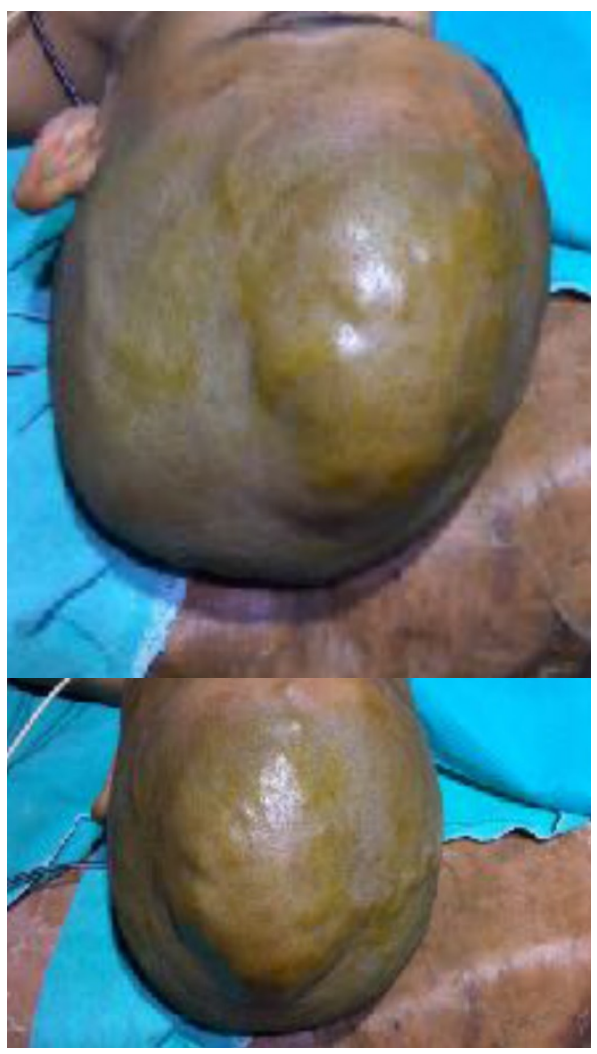

Figure 1 - Lesion over scalp 


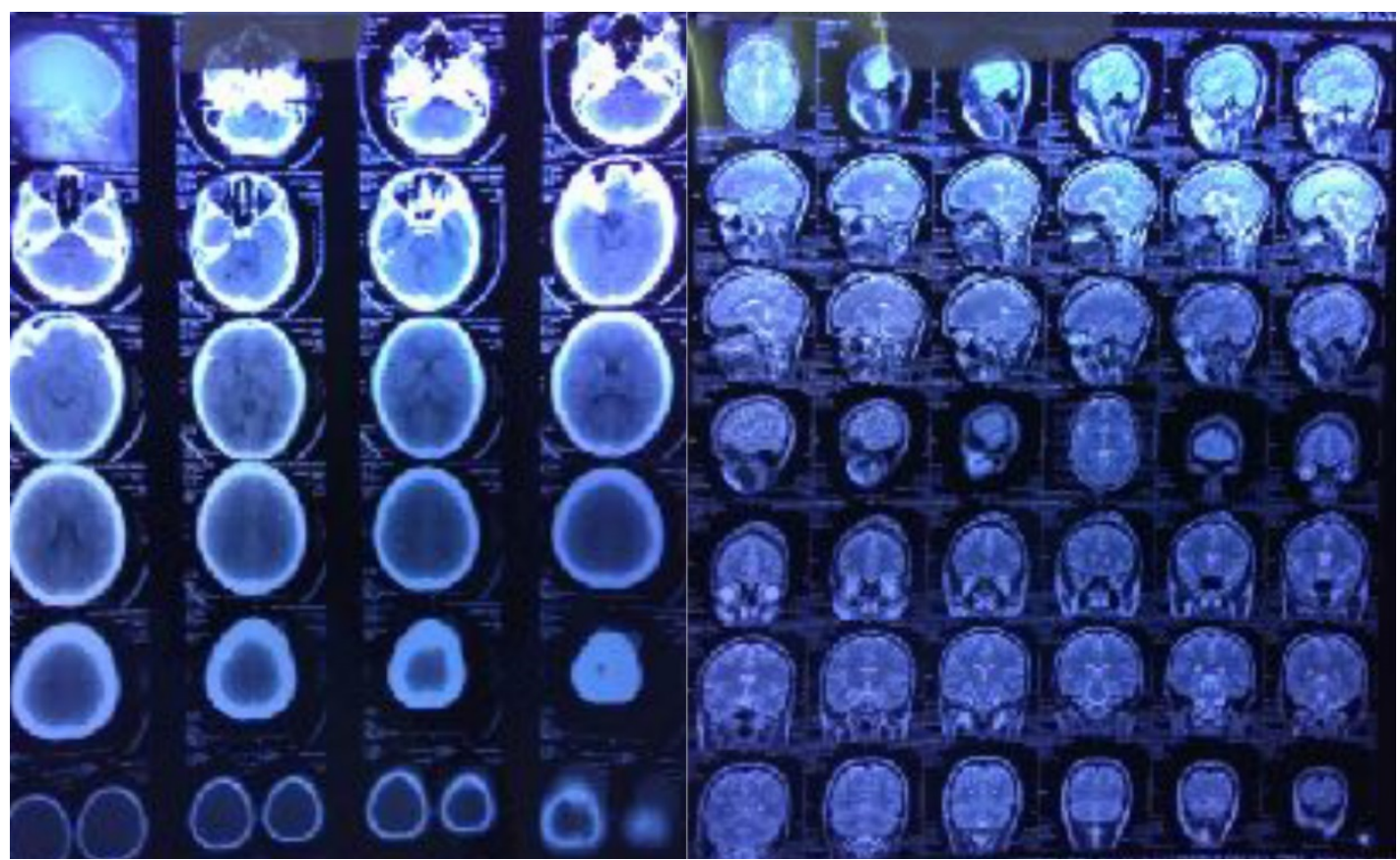

Figure 2 - MRI Brain with axial, saggital and coronal cuts with no intracranial pathology

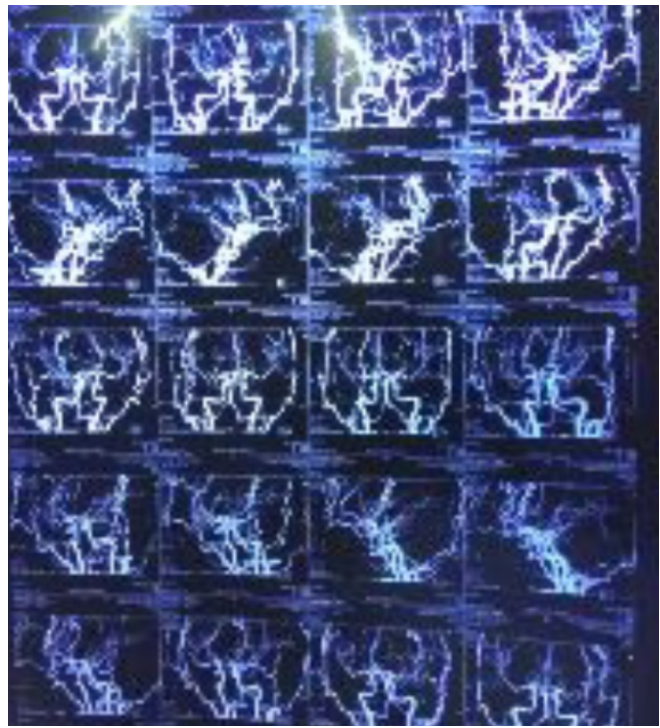

Figure 3 - MR Angiography showed high flow vascular malformation comprises multiple tortuous and dilated extra cranial arcade in the scalp particularly at the high frontal and vertex region with feeders coming from the branches of bilateral superficial temporal, occipital arteries, branches of external carotid arteries and supraorbital arteries, branches of facial arteries forming small nidus and vinous drainage in to scalp veins 
Spontaneous AVM of the scalp may present at birth, but in most patients, it is asymptomatic until adulthood. Traumatic AVM of the scalp develops months or years after the scalp trauma. About 10 to $20 \%$ of scalp AVMs develop following penetrating or non-penetrating trauma to the scalp.

Clinical signs are associated with the size of the AVM. The patients may present with headache, numbness, and/or hemorrhage. Others may present with severe symptoms such as scalp lesions. Hemorrhage is generally uncommon and may develop in the event of large vascular malformations.

Recurrent hemorrhage, which rapidly deteriorates the neurological table, may be seen in some of the patients. (15)

Most of the patients reported in the literature had a history of progressive increase in the size of the lesion and had become symptomatic in the third decade of life. $(10,16)$

Angiography is the gold standard investigation to delineate the lesion and to exclude an intracranial component.(17)

Management of scalp arteriovenous malformation is difficult because of its high shunt flow, complex vascular anatomy and cosmetic problems. The indication of treatment includes cosmetic relief of the pulsatile mass, prevention of hemorrhage and other symptoms such as headache and tinnitus.

The treatment options include surgical excision, (2) ligation of feeding vessels, transarterial and transvenous embolization, (3 4) injection of sclerosant into the nidus (5 6) and electro thrombosis. (7 8)

Surgical excision is the most common and successful method of dealing with scalp arteriovenous malformation. (10, 16)Various techniques have been used to control the hemorrhage during surgery including percutaneous sutures of the feeding vessels, interlocking suture along the line of incision, and use of tourniquet and intestinal clamp over the base of the flap. (18) A step-wise incision as suggested by other authors with careful pressure control is a useful method to control the scalp bleeding. We reflected the pericranium along with the scalp flap to prevent inadvertent rupture of the nidus. Contrary to some reports, we did not find any significant pericranial component of the malformation. As scalp AVM has a potential to evolve, the anomalous arteriovenous communication must be completely eliminated because recurrence or enlargement is reported after an incomplete treatment. Incomplete treatment can also cause scalp necrosis and bleeding. We have used a wide based scalp flap including uninvolved scalp arteries and ligation of the feeding artery near the nidus to prevent scalp necrosis.

Kumar Et al series of 31 patient concluded preoperative embolisation with cyanoacrylate or sclerosent agent followed by surgical excision gives better results compare to direct excision. Preoperative embolisationn reduses vascularity, and helps in easy identification of AVM during surgery thus achieving complete excision.

Despite these treatments, recurrence due to feeding collaterals may develop.

The most important step is total surgical excision without causing scalp necrosis and excessive blood loss. Furthermore, a better cosmetic result may be obtained. 


\section{Conclusion}

The ultimate aim of scalp AVM treatment is to give the patient's comfort by eliminating the sypmtoms. Treatment options include surgery, endovascular/percutaneous embolization, electrothrombosis, and combined approaches.

Definitive treatment may be provided with embolization in those patients with appropriate angiographic characteristics. In the event of scalp necrosis and excessive blood loss, total excision is the treatment of choice.

\section{Correspondence}

Dr. Asheesh Kumar Gupta M.Ch. resident

Department of Neurosurgery

G.R. Medical College Gwalior.

Adress- Room no. 39 Senior Boys Hostel

G.R. Medical College Gwalior

Mobile No.- 7828832632

Email-asheesh_gsvm@yahoo.com

\section{References}

1. Hasturk AE, Erten F, Ayata T; Giant non- traumatic arteriovenous malformation of the scalp. Asian Journal of Neurosurgery. 2012; 7(1): 39-41.

2. Domingo Z, Fisher-Jeffes ND, deVilliers JC. Surgical management of arterio- venous malformations of the scalp. In Schmidek HN, editor. Operative neuro- surgical techniques: Indications, methods and results. 4th Ed. Philadelphia: Saunders Company; 2000. p. 1331-8.

3. Nagasaka S, Fukushima T, Goto K, Ohjimi H, Iwabuchi S, Maehara F. Treat- ment of scalp arteriovenous malformation. Neurosurgery 1996;38:671-7.

4. Barnwell SL, Halbach VV, Dowd CF, Higashida RT, Hieshima GB. Endovascular treatment of scalp arteriovenous fistulas associated with a large varix. Radiology 1989;173:533-9.

5. Mourao GS, Hodes JE, Gobin YP, Casasco A, Aymard A, Merland JJ. Cura- tive treatment of scalp arteriovenous fistulas by direct puncture and embolization with absolute alcohol. Report of three cases. J Neurosurg 1991;75:634-7.

6. Hendrix LE, Meyer GA, Erickson SJ. Cirsoid aneurysm treatment by percuta- neous injection of sodium tetradecyl sulfate. Surg Neurol 1996;46:557-60.

7. Gardner AMN, Stewart IA. Treatment of arteriovenous malformation by en- darterial electrocoagulation. Br J Surg 1972;59:146-8.

8. Heilman CB, Kwan ES, Klucznik RP, Cohen AR. Elimination of a cirsoid an- eurysm of the scalp by direct percutaneous embolization with thrombogenic coils. Case report. J Neurosurg 1990;73:296-300.

9. Senoglu M, Yasim A, Gokce M, Senoglu N. Nontraumatic scalp arteriovenous fistula in an adult: Technical report on an illustrative case. Surg Neurol. 2008;70:194-7.

10. Muthukumar N, Rajagopal V, Manoharan AV, Durairaj N. Surgical management of cirsoid aneurysms. Acta Neurochir (Wien) 2002;144:349-56.

11. Shenoy SN, Raja A. Scalp arteriovenous malformations. Neurol India. 2004;52:478-81.

12. Massimi L, De Bonis P, Esposito G, Novegno F, Pettorini B, Tamburrini G, et al. Vertex scalp mass as presenting sign of a complex intracranial vascular malformation. J Neurosurg Pediatr. 2009;3:307-10.

13. Heilman CB, Kwan ES, Klucznik RP, Cohen AR Elimination of a cirsoid aneurysm of the scalp by direct percutaneous embolization with thrombogenic coils.Case report. J Neurosurg. 1990;73:296-300.

14. Burrus TM, Miller GM, Flynn LP, Fulgham JR, Lanzino G. NeuroImages. Symptomatic left temporal arteriovenous traumatic fistula. Neurology. 2009;73:570. 15. Fisher-Jeffes ND, Domingo Z, Madden M, de Villiers JC. Arteriovenous malformations of the scalp. Neurosurgery. 1995;36:656-60.

16. Khodadad G. Arteriovenous malformation of the scalp. Ann Surg 1973;177:79-85.

17. Wilkinson HA. Recurrence of vascular malformation of the scalp 18 years following excision. Case report. J Neurosurg 1971;34:435-7.

18. Hochberg J, Ardenghy M, Pait TG. Scalp tourniquet. Br J Plast Sur 1994;47:194-8. 